Revista de Investigación Educativa 25

julio-diciembre, 2017 | ISSN 1870-5308 | Xalapa, Veracruz

Instituto de Investigaciones en Educación | Universidad Veracruzana

\title{
Caracterización de estudiantes exitosos: Una aproximación al aprendizaje de las Ciencias Naturales
}

\author{
Characterization of successful students: An approach to the \\ learning of the Natural Sciences
}

Mtro. José Luis Olivo Franco

Docente

Técnica Agrícola Juan Domínguez Romero de Caracolí, Malambo, Colombia joseolivofranci@hotmail.com

En este artículo se describen los hallazgos de la investigación titulada "Caracterización de estudiantes exitosos: Una aproximación al aprendizaje de las Ciencias Naturales”, resumidos en tres componentes imbricados entre sí: componentes motivacionales intrínsecos, motivacionales extrínsecos, y características y estrategias de aprendizaje. La investigación se desarrolló desde un paradigma interpretativo, bajo el método fenomenológico; empleando técnicas como observación, entrevista a profundidad y grupo focal. Como muestra teórico estructural no estadística se seleccionaron intencionalmente diez estudiantes con rendimientos académicos sobresalientes de la Institución Educativa Técnica Alberto Pumarejo de Malambo, Colombia. Los procesos de categorización y microanálisis permitieron que emergieran no sólo estos tres componentes, sino también comprender la autorregulación como un fenómeno multidimensional y contextualizado, empleado por los estudiantes para tener éxito. Finalmente se prioriza sobre las actuaciones de los docentes en el uso de estrategias creativas y diversas que fomenten y desarrollen actitudes metacognitivas y autorregulatorias en los estudiantes.

Palabras clave: Motivación; metacognición; autorregulación. 
This article describes the findings of the research entitled "Characterization of successful students: An approach to the learning of the Natural Sciences", summarized in three components interwoven with each other: intrinsic motivational components, extrinsic motivational, and learning characteristics and strategies. The research was developed from an interpretative paradigm, under the phenomenological method, using techniques such as observation, in-depth interview and focus group. As a non-statistical structural theoretical sample, ten students with outstanding academic performance of the Alberto Pumarejo Technical Educational Institution of Malambo, Colombia were intentionally selected. The processes of categorization and microanalysis allowed to emerge not only these three components, but to understand self-regulation as a multidimensional and contextualized phenomenon, used by students to be successful. Finally, it is prioritized on the actions of teachers in the use of creative and diverse strategies that promote and develop metacognitive and self-regulatory attitudes in students.

Keywords: Motivation; metacognition; self-regulation.

\title{
Caracterización de estudiantes exitosos: Una aproximación al aprendizaje de las Ciencias Naturales
}

\author{
Characterization of successful students: An approach \\ to the learning of the Natural Sciences
}

\section{Introducción}

$\mathrm{L}$

as líneas de investigación de diversos autores en el campo de la enseñanza de las Ciencias Naturales se han enfocado por bastante tiempo en los errores conceptuales, las ideas previas o las dificultades de los estudiantes en el aprendizaje. Sin embargo, en este estudio se plantea como propósito comprender las características metacognitivas, autorregulatorias y motivacionales que influyen en los logros académicos de los estudiantes exitosos, desde las subjetividades de los mismos, adviértase.

En este orden de ideas, se propone un diálogo en torno a los hallazgos relacionados con la investigación "Caracterización de estudiantes exitosos: Una aproximación 
al aprendizaje de las Ciencias Naturales", que tuvo como propósito no sólo describir las características de los estudiantes exitosos, sino comprender las estructuras esenciales, el éidos de este fenómeno (Martínez, 2011). El estudio permitió interpretar que en el éxito de los estudiantes de noveno grado de la Institución Educativa Técnica Comercial Alberto Pumarejo de Malambo, Colombia, convergen múltiples factores, que se han categorizado en tres importantes aspectos imbricados entre sí: aspectos intrínsecos, aspectos extrínsecos, y características y estrategias de aprendizaje.

Es importante aclarar que no es objeto de este artículo realizar una densa revisión teórica sobre el éxito académico, sino, a partir de la información recabada, ofrecer una percepción que dé cuenta de cómo fueron develadas las características en los estudiantes seleccionados y las interacciones entre los componentes motivacionales y los constructos autorregulación y metacognición, desde las voces de los propios participantes. Con esto en mente se planteó un diálogo entre los hallazgos develados cargados de las subjetividades de los informantes y los autores consultados.

\section{Teorías referenciales}

A continuación se desarrollan de forma breve las bases teóricas de los conceptos centrales del estudio: motivación, metacognición y autorregulación; dentro de estas teorías se pueden señalar autores como Flavell, Brown, Zimmerman y Pintrich, quienes, según lo registra Lanz (2006), ofrecen planteamientos que permiten establecer:

tres tipos de relaciones entre los términos autorregulación y metacognición: 1) tratarlos como sinónimos; en estos casos el alcance real que se les asigne suele correr, principalmente, por cuenta del lector; 2) incluir el término regulación como componente de la metacognición — siguiendo la tradición de Flavell y Brown que comprende tanto la dimensión de conocimiento como de regulación-; en estos casos, se usa el termino autorregulación para referir a la dimensión autorregulativa de la metacognición; 3) subsumir ambos conceptos en el constructo del aprendizaje autorregulado, la línea de Zimmerman, Boekaerts, Pintrich, entre otros, que resulta la elaboración hasta ahora última y más comprehensiva. (p. 4)

De lo anterior se deduce que para algunos autores los límites entre metacognición y autorregulación son difusos y no muy claros, "dada la complejidad de relación entre estos términos" (García, 2012, p. 206), o como lo señalan Rosario et al. (2014), "no es posible explicarlo correctamente" (p. 782). Ahora bien, aunque hay diversas teorías 
al respecto, desde las posiciones del presente estudio se comparte la perspectiva fenomenológica de Zimmerman (2002), en la cual se considera que los aprendices que autorregulan sus aprendizajes monitorean su comportamiento en términos de sus objetivos y reflexionan sobre su progresiva eficacia. En consecuencia, mejoran su autosatisfacción y motivación, y con ello, sus métodos de aprendizaje. Desde esta arista conceptual el proceso de aprendizaje es definido por Zimmerman (2002) "como un proceso que los estudiantes hacen por sí mismos de manera proactiva y no como un evento que les sucede en reacción de la enseñanza” (p. 65).

De los planteamientos previos se deduce que el constructo conceptual de autorregulación abarca, sin duda, mecanismos, habilidades y procesos sumamente complejos. Por ejemplo, la autorregulación está ligada a procesos de carácter intrínseco como la motivación; de hecho el self es un concepto que se relaciona a su vez con dos constructos motivacionales: autoconcepto y autoestima. Y son varios los autores que dan cuenta de la interacción entre la autorregulación, la metacognición y los componentes motivacionales tanto de carácter intrínseco, como extrínseco (Berger, 2012; Boekaerts, 1999; Efklides, 2009; Núñez, Amieiro, Álvarez, García \& Dobarro, 2015; Pintrich, 1999; Ryan \& Deci, 2000; Zimmerman, 2002). Al respecto, Efklides (2009) señala que "la metacognición, la motivación y el afecto son componentes del modelo de aprendizaje autorregulado que interactúan entre sí” (p. 1). Por su lado, Berger (2012) agrega que una extensa literatura muestra que el aprendizaje autorregulado "constantemente se relaciona con numerosas creencias motivacionales” (p. 37).

Ahora bien, dentro de los primeros aportes sobre metacognición se pueden rastrear los trabajos de Flavell (1976), quien la describe como el "conocimiento que uno tiene acerca de los propios procesos y productos cognitivos o cualquier asunto relacionado con ellos" (p. 232). Otros, afirman Ellis, Denton y Bond (2014), “aceptan básicamente la descripción de Flavell, ampliando el término para abarcar actividades cognitivas como la reflexión, la sensibilidad, la autorregulación, la autoevaluación e incluso la función agentiva” (p. 4016). Precisando aún más, Efklides (2009) muestra que la metacognición "contribuye a la autorregulación del aprendizaje a través de múltiples vías basadas justamente en el control de la cognición” (p. 80).

Sin embargo, desde la teoría social-cognitiva, Zimmerman (2002) apunta que la metacognición hace parte del constructo multidimensional del aprendizaje autorregulado, y "aunque por sí sola es insuficiente cuando se carece de habilidades fundamentales, es una disposición esencial para la autorregulación” (p. 65), ejerciendo un 
papel clave en el monitoreo y control de la cognición. En este sentido, el aprendizaje autorregulado se muestra como un proceso de origen social donde los planteamientos vygotskianos se pueden rastrear; es un proceso por medio del cual un estudiante fija sus propias metas, monitorea, controla y regula la adquisición de su cognición (metacognición), emociones, motivación y conducta, orientado por metas y características contextuales e interacciones con el entorno.

Se puede referenciar también a Bandura (1991), quien esboza en su Teoría Social Cognitiva la forma como operan las estructuras del sistema autorregulatorio concretadas mediante tres subprocesos: auto-observación, autojuicios y autorreacciones. En esta línea, Zimmerman (2002) describe un modelo explicativo cíclico del desarrollo de la autorregulación a través de tres fases y subprocesos: fase de previsión (forethougth), fase de control de desempeño (performance control) y fase de autorreflexión (self-reflection). Igualmente, este modelo abarca cuatro niveles de desarrollo: observación, emulación, autocontrol y autorregulación, siendo los dos primeros consecuencia de la influencia social, en contraste con los siguientes, en los cuales la influencia se desplaza hacia el sujeto como aprendiz.

\subsection{Motivación}

El papel de la motivación en el aprendizaje autorregulado ha sido estudiado por varios autores, entre quienes sobresalen Pintrich (1999), Ryan y Deci (2000), Yamac y Ocak (2013), entre otros. Inicialmente, Pintrich (1999) presenta un marco general para ayudar a entender la relación entre la motivación y el aprendizaje autorregulado; según este marco, el aprendizaje autorregulado puede facilitarse por la adopción de metas de dominio y capacidad relativa, y obstaculizarse por la adopción de objetivos extrínsecos. Igualmente señala que algunas variables motivacionales que afectan el aprendizaje autorregulado son: el contexto sociocultural (normas, cultura y prácticas educativas), el contexto de la clase (la tarea, la autoridad, el reconocimiento, la dimensión grupal, la evaluación), los factores internos (las creencias y percepciones, metas y emociones) y la conducta motivada (la elección conductual, el nivel de la actividad y de compromiso, la persistencia y control del esfuerzo, entre otras).

Por otra parte, Ryan y Deci (2000), en su teoría de la autodeterminación ofrecen un enfoque "que enfatiza la importancia de la evolución de los recursos humanos internos para el desarrollo de la personalidad y la autorregulación de la conducta” (p. 2). Con este propósito examinaron "factores que amplían versus que reducen la motiva- 
ción intrínseca, la autorregulación y el bienestar” (p. 1). Sus hallazgos los condujeron a postular tres necesidades psicológicas innatas: competencia, autonomía y relacionarse, las cuales, cuando son satisfechas, producen ampliación de la automotivación y la salud mental, y cuando son frustradas conducen a la reducción de la motivación y el bienestar. Así mismo, Weiner (2000) señala aspectos intrapersonales e interpersonales del sistema motivacional, cuyos "límites entre uno y otro son difusos, pero vale la pena hacer la distinción" (p. 2).

De otro lado, Efklides (2009) muestra las relaciones entre metacognición y motivación; por ejemplo, la decisión de continuar o no una tarea e invertir esfuerzo en ello, depende de la conciencia metacognitiva, de las características de la tarea, de las habilidades metacognitivas de la persona y de su interacción con la tarea y el contexto. Por tanto, hay dos maneras en que la metacognición puede influir en la motivación y por medio de ella lograr la autorregulación del comportamiento; primero, a través de sus efectos sobre los propios procesos del aprendiz y, segundo, a través de sus efectos sobre las atribuciones causales, específicamente, experiencias metacognitivas. Finalmente, concluye Efklides (2009), es la interacción de todas las facetas de la metacognición la que desempeña un papel importante y particular, la motivación inducida por las experiencias metacognitivas, que activa el conocimiento metacognitivo y/o las habilidades metacognitivas existentes.

Llegados a este punto es evidente que la perspectiva sociocognitiva posibilita un planteamiento teórico conceptual abarcador, que permite abordar otros factores de la cognición y la motivación intrínsecos y extrínsecos (Weiner, 1985), y en esa medida tener un referente en cuanto a la caracterización del éxito académico de los estudiantes se trate.

\section{Método}

Vale la pena señalar que para orientar el proceso de investigación se plantearon interrogantes como: ¿Cuáles son, según los estudiantes de noveno grado de la Institución Educativa Técnica Alberto Pumarejo de Malambo, Colombia, las características metacognitivas, autorregulatorias y motivacionales que influyen en su éxito académico en Ciencias Naturales?, ¿qué relación se puede establecer entre las características de los estudiantes exitosos de noveno grado, participantes en el estudio, y la información aportada por la bibliografía especializada? 
Es evidente que la naturaleza de los interrogantes formulados enfocó la investigación hacia la comprensión de las características de los estudiantes exitosos y, en este sentido, tal como lo reconoce Martínez (2011), "la lógica tradicional resulta incapaz de comprender los complejos problemas de este fenómeno propio de las ciencias sociales, de las realidades humana, y los conceptos derivados de éstas no se pueden reducir a términos cuantitativos inesenciales y aconceptuales" (p. 79). Por consiguiente, el trabajo se desarrolló desde un enfoque interpretativo-dialéctico (Martínez, 2011), en tanto que este paradigma, según Rojas (2014), "ha permitido hacer más próximos los resultados de la investigación a la realidad educativa” (p. 13). El método fenomenológico posibilitó, como diría Husserl (1974), "ir hacia las cosas mismas" (p. 233), develando la estructura del fenómeno del estudiante exitoso, tal y como se presenta desde las subjetividades de los actuantes.

Aproximarse a los propósitos planteados demandó delimitar qué se entiende por estudiantes exitosos. Para ser coherentes y congruentes con la naturaleza fenomenológica de la investigación, se tuvieron en cuenta hallazgos de un estudio inicial titulado "Aproximación interpretativa de docentes sobre estudiantes exitosos", que permitió develar la caracterización de un estudiante exitoso desde la mirada de siete docentes de Ciencias Naturales de Malambo entrevistados. ${ }^{1}$ Dada la naturaleza eminentemente fenomenológica del trabajo, se decidió presentar una concepción de estudiante exitoso basada en un juego de interacciones entre las miradas de los docentes y los autores.

A continuación se expondrán brevemente las conclusiones a las que se llegaron en el estudio: los docentes hicieron énfasis en aspectos motivacionales extrínsecos e intrínsecos, actitudes cognitivas, y se refirieron implícitamente a actitudes metacognitivas y autorregulatorias. Pero de forma interesante enfatizaron que un estudiante exitoso se reconoce por su gusto e interés por aprender. Dejan claro, además, que el apoyo de los padres en los procesos de formación de sus hijos es una característica común que favorece el éxito académico. Sólo un docente señaló la obediencia como un rasgo distintivo y deseado de los estudiantes exitosos. No se señalaron datos concretos y significativos que den cuenta de la edad o el momento en que los estudiantes adquieren actitudes relacionadas con la metacognición o la autorregulación, y mucho menos si éstas son innatas o heredadas, aprendidas o desarrolladas. En la Tabla 1 se muestra un resumen de las principales características que identifican a un estudiante exitoso según los docentes entrevistados.

1. El guión de la entrevista aplicada a los docentes se basó en el estudio de Gómez (2003). 


\section{Tabla 1. Aproximación interpretativa de docentes sobre estudiantes exitosos}

\begin{tabular}{|c|c|c|c|}
\hline Pregunta & Propósito especifico & Categorías & Subcategorías \\
\hline $\begin{array}{l}\text { Cómo profesor(a) de una } \\
\text { institución ha visto pasar } \\
\text { a estudiantes brillantes } \\
\text { y otros que necesitan } \\
\text { ayuda, imagine, por } \\
\text { favor, que puede duplicar } \\
\text { el ingreso del primer } \\
\text { grupo a sexto grado y } \\
\text { separar de aquellos que } \\
\text { requieren de más apoyo: } \\
\text { ¿en qué se basaría para } \\
\text { formar los grupos?: } \\
\text {-Brillantes } \\
\text {-Los que necesitan de } \\
\text { apoyo }\end{array}$ & $\begin{array}{l}\text { Develar las } \\
\text { características que } \\
\text { según los docentes de } \\
\text { Ciencias Naturales de } \\
\text { Malambo identifican a } \\
\text { los estudiantes exitosos } \\
\text { y factores que inciden } \\
\text { en el éxito de estos } \\
\text { estudiantes. }\end{array}$ & $\begin{array}{l}\text { Características de } \\
\text { estudiantes exitosos } \\
\text { Características de la } \\
\text { interacción }\end{array}$ & $\begin{array}{l}\text { Pasión y gusto por } \\
\text { aprender. } \\
\text { Dinámicos } \\
\text { Sujetos reflexivos } \\
\text { Autocontrol } \\
\text { Líderes } \\
\text { Autoconocimiento } \\
\text { Motivación familiar } \\
\text { Entorno y } \\
\text { compañeros. }\end{array}$ \\
\hline $\begin{array}{l}\text { ¿Qué tienen en común } \\
\text { los alumnos que } \\
\text { consideraría como } \\
\text { brillantes? }\end{array}$ & & & \\
\hline $\begin{array}{l}\text { ¿Algún comportamiento } \\
\text { especial en el aula? } \\
\text { Si tuviese que elegir a } \\
\text { sus propios estudiantes } \\
\text { o con la ayuda de } \\
\text { otras personas: ¿qué } \\
\text { características desearía } \\
\text { que tuvieran? } \\
\text { ¿Qué preguntas haría } \\
\text { para saber que tienen } \\
\text { esas características? }\end{array}$ & $\begin{array}{l}\text { Analizar qué aspectos } \\
\text { comportamentales } \\
\text { consideran los docentes } \\
\text { de Ciencias Naturales } \\
\text { de Malambo que deben } \\
\text { tener los estudiantes } \\
\text { exitosos. } \\
\text { Conocer qué estrategias } \\
\text { de aprendizaje usan, } \\
\text { según los docentes } \\
\text { de Ciencias Naturales } \\
\text { de Malambo, sus } \\
\text { estudiantes exitosos. }\end{array}$ & $\begin{array}{l}\text { Comportamientos } \\
\text { exhibidos por } \\
\text { estudiantes exitosos } \\
\text { Estrategias }\end{array}$ & $\begin{array}{l}\text { Curiosos } \\
\text { Disciplinados } \\
\text { Obedientes } \\
\text { Empáticos } \\
\text { Competencias } \\
\text { verbales y discursivas } \\
\text { Capacidad para } \\
\text { resolución de } \\
\text { problemas } \\
\text { Propositivos } \\
\text { Inquietos } \\
\text { Sarcásticos respecto } \\
\text { a la realidad } \\
\text { Profundizan } \\
\text { No se conforman } \\
\text { Perspicaces } \\
\text { Discernidores }\end{array}$ \\
\hline
\end{tabular}

Fuente: Elaboración propia. 
Posteriormente, se focalizaron las características evidenciadas por el estudio inicial, y luego se definió la muestra de estudio, así como las formas de recolectar información, el diseño y aplicación de técnicas o instrumentos, seleccionando la entrevista y el grupo focal para tal propósito, y siguiendo con la organización y análisis de los hallazgos.

Se debe aclarar que la representatividad de la muestra no radica en la cantidad de la misma, sino en las posibles configuraciones subjetivas de los sujetos con respecto al éxito académico. Partiendo de estos supuestos como fundamentos metodológicos, epistémicos y pragmáticos, se afirma en primera instancia que la población de esta investigación la constituyen los estudiantes de educación básica secundaria de la Institución Educativa Alberto Pumarejo. Sin embargo, hay que especificar que la muestra la conforman diez estudiantes de noveno grado con un destacado rendimiento académico. Adviértase que se trata de una muestra teórica estructural no estadística, hecho que "permite abordar desde lo intensivo las características de los fenómenos, desentendiendo su generalización cuantificable y extensiva” (Serbia, 2007, p. 132).

El proceso se describe a continuación. En primera instancia, se indagó sobre las instituciones educativas públicas del municipio cuyos estudiantes han demostrado éxito académico durante los últimos años. Para ello, se consultaron principalmente los resultados de las Pruebas Saber en Ciencias Naturales (Instituto Colombiano para el Fomento de la Educación Superior [ICFES], 2016). ${ }^{2}$ Esta primera indagación pudo dar cuenta de la Institución Educativa Técnica Comercial Alberto Pumarejo (IETCAP), escuela pública que en los últimos años se ha destacado por los resultados de sus estudiantes en varios ámbitos, entre estos, el académico, a nivel municipal, regional y nacional.

Se procedió luego a seleccionar a los estudiantes de noveno grado de esta escuela que durante su vida escolar han demostrado ser exitosos, con la colaboración del departamento de orientación del plantel, que facilitó una lista de estudiantes destacados en todos los cursos de noveno grado, ${ }^{3}$ basado en los reportes académicos y convivenciales del año anterior (2015) y del primer trimestre de 2016. Del tal manera, se tuvo un grupo de veinticuatro estudiantes, de los cuales se seleccionaron diez, teniendo como criterio su promedio académico y disponibilidad.

2. Las Pruebas Saber son evaluaciones de estado aplicadas anualmente por el ICFES a estudiantes de educación básica primaria y secundaria.

3. El noveno grado corresponde al último grado de la educación básica secundaria reglamentado por el artículo 19 de la Ley 115 de 1994, o Ley General de Educación en Colombia. 
Por otro lado, los aportes de Strauss y Corbin (2002) contribuyeron a la constitución de categorías a partir de un ejercicio de microanálisis de los datos obtenidos mediante la entrevista y el grupo focal, siguiendo las sugerencias de Coffey y Atkison (2003). Se desarrollaron diez entrevistas a sendos estudiantes, teniendo como criterios de selección: la saturación teórica (Creswell, 1994; Vasilachis, 2006) y la disposición de los informantes (relacionado con la voluntad y disposición personal y de horarios concedidos por la escuela para realizar el trabajo). ${ }^{4}$ Una vez que se dio lectura a los textos de las entrevistas, con el fin de confirmar o ampliar algunos aspectos relacionados con los primeros hallazgos, se procedió a desarrollar el grupo focal, organizándose en tres sesiones de tres, cuatro y tres participantes cada una; el tema específico a tratar giró en torno a los aspectos motivacionales y autoregulatorios de los participantes. Todo ello bajo consideraciones de credibilidad, confirmación, significado en contexto, patrones recurrentes y consideraciones éticas que autores como Hernández, Fernández y Baptista (2010) y Rojas (2014) señalan.

El análisis de la información se llevó a cabo tomando como base el modelo de análisis propuesto por Strauss y Corbin (2002). El proceso de codificación se hizo en tres líneas: abierta, axial y selectiva, lo cual supuso un microanálisis línea por línea de cada una de las entrevistas y sesiones del grupo focal, fragmentando los textos e identificando las ideas más importantes que aludían a diferentes aspectos con colores específicos (cromatización).

Para favorecer el proceso de análisis, fragmentación de textos y configuración de categorías y subcategorías se asignó a cada participante un código, sustituyendo sus nombres por una nomenclatura. ${ }^{5}$ Igualmente es necesario señalar que a partir de códigos "in vivo" (esto es, desde el lenguaje y expresiones propias de los estudiantes), se codificaron en un primer momento las subcategorías; este proceso correspondió a la codificación axial. Para la codificación selectiva las subcategorías que compartían

4. Tanto las entrevistas, como el grupo focal se desarrollaron durante las horas de clase destinadas a la asignatura de Ciencias Naturales, previo permiso del rector de la institución, de la docente titular, y la socialización a estudiantes de noveno grado, en un espacio cómodo, evitando interrupciones y con una duración aproximada de quince a veinte minutos.

5. Esta nomenclatura se utilizará a lo largo del trabajo para anonimizar la identidad de los participantes; consta de literales y números: las letras Ex se refieren a estudiante exitoso; el 9 indica el grado noveno; los siguientes dos números indican el curso específico al que pertenecen (01, 02, 03, 04); el último es el número asignado al participante cuando se realizó la entrevista; la letras finales señalan la fuente de la información: EN, que proceden de la entrevista y GF, que provienen del grupo focal. Igualmente los nombres de los docentes han sido cambiados a fin de proteger su identidad. 
propiedades o dimensiones similares se subsumieron en categorías; a su vez, las categorías que compartían propiedades similares se subsumieron en niveles centrales llamados aspectos. Para ilustrar: las subcategorías gusto y satisfacción, expectativas y responsabilidad, se subsumieron en la categoría motivación intrínseca, y a su vez, las categorías motivación intrínseca y autoconcepto se subsumieron en un nivel central llamado aspectos intrínsecos.

\section{Hallazgos}

Apuntar a los hallazgos aquí es plantear consideraciones en un tono vívido y natural (Hernández, Fernández \& Baptista, 2010), para evitar la dureza positivista, pero presentadas con la mayor seriedad posible en torno al tópico de estudiantes exitosos, en una fluida conversación, desde las voces de los estudiantes que se harán presentes a lo largo del discurso, en un diálogo a la manera de Maturana (2002), ${ }^{6}$ con las teorías, los autores, otras investigaciones y, por supuesto, las propias ideas del sujeto cognoscente sobre el fenómeno en cuestión.

En primera instancia, fue posible atisbar algunos rasgos generales de los participantes. Son adolescentes de estrato socioeconómico bajo: uno y dos, cuyas edades oscilan entre los trece y catorce años; varios viven en un tipo de familia extendida, es decir, con abuelos o tíos, mientras que dos de ellos pertenecen a una familia nuclear, formada por sus padres o uno de sus padres. Esta radiografía general del grupo permite observar que los estudiantes exitosos viven en condiciones similares a las de los demás escolares, enfrentando los mismos problemas de orden social y político a los cuales la gran mayoría de jóvenes malamberos se enfrenta, pudiendo sugerir en una primera mirada que los altos rendimientos no dependen necesariamente del estrato social.

Ahora bien, para hablar del éxito de los estudiantes en ciencias se dejó que ellos mismos lo hicieran mediante la entrevista y el grupo focal, permitiendo la construcción de diez bitácoras que posibilitaron la comprensión y caracterización del fenómeno estudiante exitoso. Así pues, a partir del proceso de análisis se consolidaron tres aspectos centrales: aspectos internos, aspectos extrínsecos y estrategias y características de aprendizaje.

6. Según Maturana (2002, p. 46), un diálogo es el flujo continuo de trazamientos de dominios relacionales, emocionales y recurrentes coordinaciones consensuales de conducta. 


\subsection{Aspectos intrínsecos}

Los aspectos intrínsecos fueron constituidos a partir de las categorías y subcategorías que se muestran en la Tabla 2. Desde la mirada de los estudiantes, su rendimiento se relaciona sustantivamente con sus metas, sus expectativas, porque les gusta leer e investigar. El éxito es sinónimo de dedicación y esfuerzo. Uno no es exitoso porque sí, sino porque se traza su camino hacia este fin; por consiguiente, para explicar el rendimiento y el aprendizaje alcanzado por un individuo han de conjugarse componentes cognitivos, metacognitivos y afecto-motivacionales (Bandura, 1999; Núñez, Amieiro, Álvarez, García \& Dobarro, 2015; Pintrich, 1999; Zimmerman, 2002). Los constructos conceptuales que emergieron desde la interpretación de los textos agrupados en los aspectos intrínsecos permiten consolidar lo señalado por Ryan y Deci (2000), al hacer perceptible que factores como la motivación intrínseca impulsan el aprendizaje de un individuo. De ahí que este primer componente se constituyó por las categorías motivación intrínseca y autoconcepto.

Tabla 2. Categorías y subcategorías del componente o aspectos intrínsecos

\begin{tabular}{|c|c|c|}
\hline Componentes o Aspectos & Categoría & Subcategoría \\
\hline \multirow{6}{*}{ Aspectos intrínsecos } & \multirow{3}{*}{ Motivación intrínseca } & Éxito fruto del gusto y la satisfacción \\
\hline & & Éxito fruto de la disciplina \\
\hline & & Éxito fruto de expectativas \\
\hline & \multirow{3}{*}{ Autoconcepto } & Ser responsable \\
\hline & & Sentirse orgulloso \\
\hline & & Metas \\
\hline
\end{tabular}

Fuente: Elaboración propia.

Motivación intrínseca es la primera categoría, en la que se incluyeron subcategorías como: éxito fruto del gusto y la satisfacción, éxito fruto de la disciplina, éxito fruto de expectativas. Para algunos estudiantes, su éxito se debe a que el estudio es para ellos una experiencia vital que los empuja a aprender, a comprender un tema, hacerlo les produce satisfacción, aunque el resto de sus compañeros no lo entienda. El siguiente comentario evidencia lo señalado anteriormente: "Cuando uno quiere algo tiene que 
hacer todos los esfuerzos posibles para lograrlo y ver que ha producido fruto es una gran satisfacción" (EX9015GF).

En efecto, tal como señalan Ryan y Deci (2000), la autosatisfacción es un componente importante que amplía el aprendizaje. En estos casos se trata de una motivación interna o auténtica (literalmente atribuida del yo o atribuida a este), porque se percibe en estos textos interés, excitación y confianza, lo cual se manifiesta en su persistencia, desempeño y creatividad y esto, a su vez, exhibe autorregulación del aprendizaje (Ryan \& Deci, 2000; Rosario et al., 2014; Zimmerman \& Moylan, 2009). De igual manera, los componentes motivacionales de los jóvenes sugieren uno de los factores de los componentes motivacionales de valor de una tarea: el valor intrínseco, definido como la satisfacción que obtienen las personas de su actuación o del desarrollo de una actividad en las que se experimenta el placer de aprender (Yamac \& Ocak, 2013).

Se devela otro componente intrínseco, la subcategoría autoconcepto, que va a determinar en gran medida la motivación escolar y que debería, por consiguiente, ser referencia obligada de todo profesor que desee incidir en la motivación de sus estudiantes (Ryan \& Deci, 2000). Entre los interrogantes de la entrevista que permitieron develar cuatro dimensiones del autoconcepto se plantearon: ¿Cómo te defines como persona? y ¿cómo te defines como estudiante? Se construyó una primera subcategoría, ser responsable, relacionada con la dimensión del autoconcepto académico y la llamada dimensión cognitiva, que atiende al grado de entendimiento o comprensión que se cree disponga el yo, el estudiante mismo.

Se enmarca en algunos textos, además, el factor motivacional al logro que "es la disposición a hacer mejor las cosas, a tener éxito y a sentirse competente” (Becerra \& Morales, 2015, p. 138). Por consiguiente, los motivos que impulsan a estudiar en estos casos particulares suelen ser el deseo de superación, el ser mejor, el deseo de conocer, de aprender más y de realización.

En segundo lugar, la subcategoría sentirse orgulloso señala una dimensión afectiva, relacionada estrechamente con el grado de satisfacción con uno mismo: "Me hace sentir orgullosa de mí misma por lo que he logrado y cómo he superado todas las etapas... y he llegado aquí... este, bien, por decirlo así" (EX9022EN); "Yo me siento muy feliz, orgullosa de mí misma, tanto de mi esfuerzo, como el esfuerzo que hacen mis padres por tenerme en el colegio" (EX9019EN).

En este sentido, se observa cómo el yo expresa el grado de satisfacción consigo mismo; por medio de este proceso una persona se valora positivamente (Ryan \& Deci, 2000). De esta manera, se verifica que estos estudiantes tienen una autoestima alta, 
son conscientes de que se les reconoce, les gusta que se les admire y que sean motivo de orgullo para sus padres y la institución. La tercera dimensión del autoconcepto emerge de la subcategoría metas, constituida a partir de textos como los siguientes: "Como una mujer exitosa, pero digamos no sólo en lo laboral sino en lo personal, tener una familia. O sea, ser inteligente, me gustaría como tener mi propia empresa y eso, también me interesa la medicina, quiero tener las dos cosas" (EX9022GF); "La verdad, cuando estuviera grande me gustaría estudiar Física Cuántica y eso... la seño Maige me anima. Uff, desde hace años" (EX9015GF).

El constructo metas recuerda el "yo posible" (Zimmerman, 2002), refiriéndose a la representación que construye el estudiante proyectándose al futuro; es así como expresiones "ser una mujer exitosa”, "estudiar Física”, son ejemplos de textos típicos que posibilitaron tal categorización. Ahora bien, las metas que se observan en esta categoría son aspiraciones intrínsecas, como lo señalan Ryan y Deci (2000), asociadas "positivamente con indicadores de bienestar como auto-estima, la auto-actualización y la reversión de la depresión y la ansiedad” (p. 11). En síntesis, según los relatos, el autoconcepto característico de estos jóvenes es ser personas amables, respetuosas, perseverantes, emprendedoras, capaces de resolver problemas. Demuestran, además, una sana autoestima, son conscientes de la admiración y reconocimientos que reciben por su rendimiento académico.

Las metas de los estudiantes se pueden enmarcar como un tipo situacional influenciadas culturalmente por "salir adelante", "ser alguien en la vida", "proveer una mejor situación socioeconómica a su madre”, es decir, una situación que ofrece el contexto y de tipo personal, que se motiva por elementos internos como la satisfacción, el sentido de orgullo y la autoestima (Alcántara \& Bernal, 2012; Ryan \& Deci, 2000, Weiner 2000). Adviértase, además, que algunas metas se corresponden con motivación extrínseca, en tanto se orientan a obtener mejores calificaciones, incluso hacia sobresalir por encima del otro; resulta interesante que según Ryan y Deci (2000), tales metas están negativamente relacionadas con indicadores de bienestar como la ansiedad y la depresión, confirmar esta relación o no en esta muestra resultaría de gran interés, pero tal propósito excede los alcances de este estudio.

En este hilo argumental, se señala que la motivación y la cognición, aunque son condiciones positivas que amplían el aprendizaje, no son suficientes, y deben existir mecanismos de control de los niveles de motivación; por esto, Ryan y Deci (2000) apuntan mecanismos como la autodeterminación (relacionada con la autorregulación) y Efklides (2011), por su parte, a la metacognición. Es posible representar las 
categorías y subcategorías relacionadas con los aspectos anteriores en función del sistema y control de protección constituido por la metacognición y la autorregulación, como se muestra en la Figura 1.

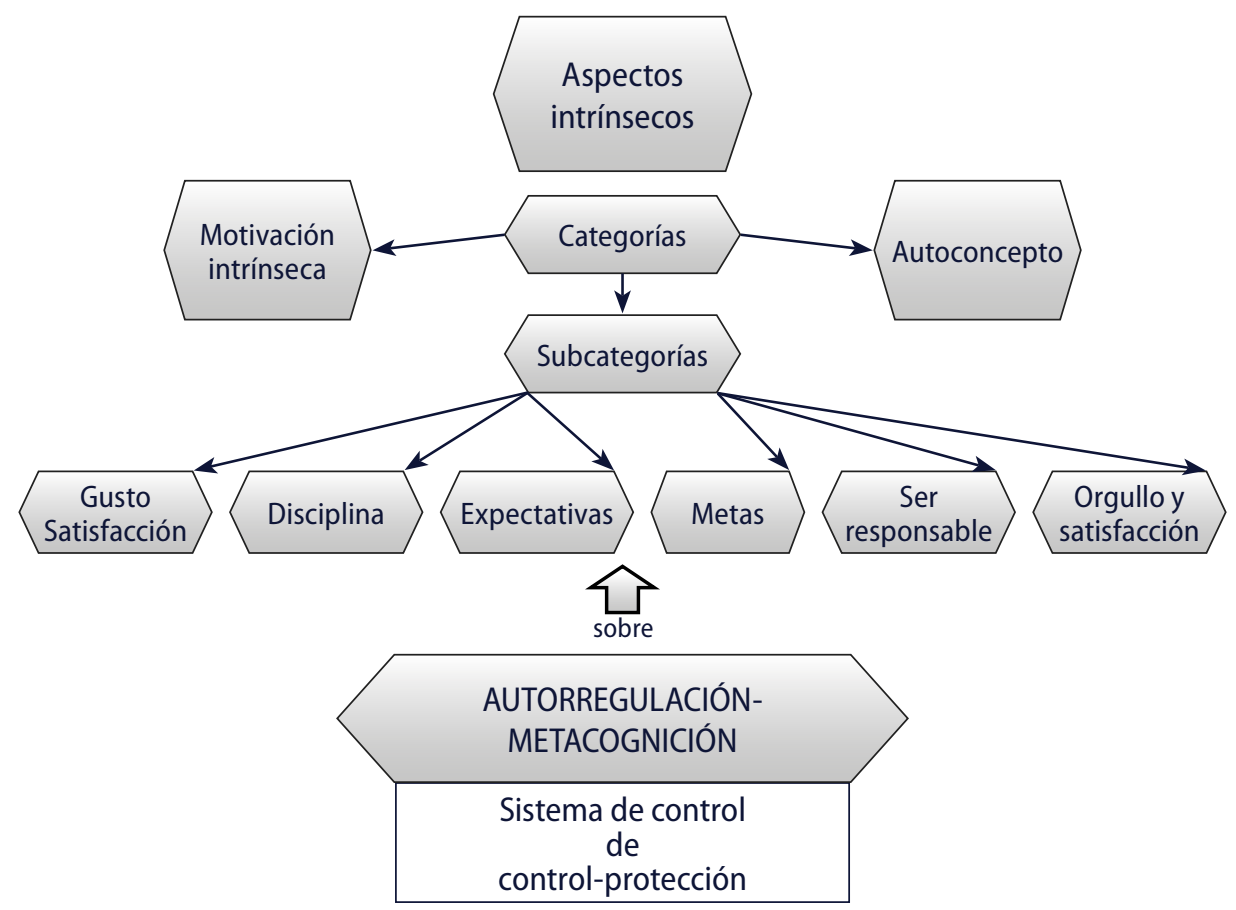

Figura 1. Categorías y subcategorías del componente o aspectos Intrínsecos en función del sistema de control y protección Autorregulación-Metacognición

Fuente: Elaboración propia.

\subsection{Aspectos extrínsecos}

Los relatos permitieron el constructo de motivación extrínseca, que alude al "desempeño de una actividad a fin de obtener algún resultado separable y, por lo tanto, contrasta con el de motivación intrínseca que se refiere al hacer una actividad por la satisfacción inherente que ocasiona la actividad por sí misma” (Ryan \& Deci, 200o, p. 6), 
y se constituyó por subcategorías como éxito fruto de la motivación familiar. En ésta aparece de forma interesante el papel de la madre como referente principal o impulsadora del éxito de los estudiantes (ver Tabla 3 ).

\section{Tabla 3. Categorías y subcategorías de aspectos o componentes extrínsecos}

\begin{tabular}{ccc}
\hline Aspectos & Categoría & \multicolumn{1}{c}{ Subcategorías } \\
\hline \multirow{3}{*}{ Aspectos extrínsecos } & Motivación extrínseca & Éxito fruto de la motivación familiar \\
\cline { 3 - 3 } & & Éxito fruto de la interacción con docentes \\
\cline { 3 - 3 } & & Éxito e influencia del entorno \\
\hline
\end{tabular}

Fuente: Elaboración propia.

En relatos como el siguiente fue posible percibir la influencia materna: "Mi familia, principalmente mi mamá, yo creo que uno de mis profesores también, que hacen que uno se supere y quiera ser mejor. O sea, yo creo que poner orgullosa a mi mamá, tenerla orgullosa de cómo me cría" (EX9022EN).

El comentario anterior explicita la influencia de los padres frente a la escolaridad de sus hijos, particularmente en entornos desfavorecidos en sentido económico, como los que viven los participantes. Al respecto, Ryan y Deci (2000) señalan estudios de madres e infantes que han demostrado, sin lugar a duda, "que tanto la seguridad como el apoyo maternal a la autonomía predicen una mayor conducta exploratoria en los infantes" (p. 5). Tales condiciones facilitan la motivación intrínseca.

En el otro extremo de las motivaciones extrínsecas aparece la influencia de sus profesores, constituyendo la subcategoría éxito fruto de la interacción con docentes:

Y unos profesores también hacen que uno se supere, quiera ser mejor. La seño Daly, el profesor Mesa y la seño Doris. Yo pienso que cada uno de ellos tiene como cierta característica que yo quiero ser a futuro o trato de meter a mi vida eso que ellos tienen. Yo definiría a la seño Daly como una dama, o sea, ella siempre se ve perfecta por decirlo así, siempre parece muy inteligente, muy feliz, entonces yo como a futuro quiero ser así. Al profesor Mesa, él es como la parte comercial del colegio, entonces es otro que me llama la atención la forma como se expresa, como un poco relajado, pero sin dejar sus responsabilidades y todo eso, eh, me gusta. La 
seño Manuela, yo quisiera ser así cuando sea grande, sabe, por decirlo así, de todo, la forma en que habla y todo lo que ha logrado hasta ahora. (EX9022GF)

Es posible percibir cómo los estudiantes reconocen el rol que desempeñan sus docentes en los procesos de formación, emerge de los textos una aproximación al perfil docente compuesta de varias dimensiones (Urbina, 2012). De acuerdo con los jóvenes, un maestro que refleja valores y actitudes de empatía, amabilidad, afecto, es exigente pero no dogmático, respetuoso, dispuesto a escuchar, logra movilizarlos hacia apasionarse por la asignatura, hacia el logro de desempeños académicos exitosos (González, 2013); lo anterior corresponde con una dimensión personal del docente. En estos casos, la influencia de los docentes actúa como motivación extrínseca vinculada a una aceptación personal y a un sentimiento de elección, en la medida que estos docentes han logrado de alguna manera la internalización (meter a su vida) de metas como superación personal en sus estudiantes (Ryan \& Deci, 2000).

La dimensión pedagógica se logra leer en los textos de los estudiantes que se refieren a cómo sus profesores interactúan con ellos en el acto pedagógico: "Explican bien, buscan la atracción para que uno entienda más, por los juegos o actividades que nos ponen como la seño Manuela, maestros que tienen gran interés en estudiar cada vez más, algo que me relaciona con ellos" (EX9022GF). La dimensión pedagógica del rol docente para este grupo de estudiantes es señalada implícitamente en sus relatos, porque sus profesores fomentan ambientes de aprendizaje agradables, capaces de despertar empatía y gusto por la asignatura impartida y por los estudios (González, 2013).

En tercer lugar, hay una dimensión vocacional referida no sólo a manifestar entusiasmo, sino también llevarlo a cabo de manera inteligente. De igual forma, la dimensión de expectativas se constituye en relatos que evidencian que estos docentes generan para sus estudiantes altas expectativas, les animan a encaminar sus esfuerzos para que puedan lograrlas progresivamente: "La seño Manuela, su manera de explicar me encanta, la verdad cuando yo sea grande me gustaría estudiar física cuántica y eso la seño Manuela me anima." (EX905EN).

Finalmente emerge una dimensión interpersonal relacionada con el docente, en esencia se refiere a las relaciones que establecen los profesores con sus estudiantes. Los estudiantes exitosos sienten empatía hacia estos docentes que están dispuestos a escucharles, orientarles, dedicarles tiempo aclarando sus dudas: "Yo digo que también los profesores nos ayudan [...] porque nos orientan, ellos no nada más nos explican las clases y hagan esto, tareas, por ejemplo; nos orientan, [si] tenemos problemas, nosotros podemos decirles a ellos" (EX9018GF). 
Que los estudiantes expliciten el hecho de que los docentes tienen que ver de alguna manera motivacional con su éxito académico, puede indicar que este grupo de docentes ha comprendido que la motivación es un fenómeno multifacético y dinámico, en contraste con la idea tradicional que asume que los estudiantes deben ser exitosos estén motivados o no, y desconocer que éstos puedan ser motivados de diferentes maneras (Pintrich, 1999; Alcántara \& Bernal 2012) y da bases para inferir que tales docentes movilizan estrategias y recursos a fin de lograr motivar a sus alumnos. Podría resumirse que tanto la influencia de la familia como de los docentes ha logrado cierta motivación identificada, "asociada con un mayor interés y disfrute de la escuela y estilos de afrontamiento más positivos, así como con el empleo de mayores esfuerzos” (Ryan \& Deci, 2000, p. 9).

Por último, la subcategoría éxito e influencia del entorno, revela cómo los compañeros y el contexto juegan un papel destacado en la vida social y académica para estos jóvenes, estableciendo relaciones especialmente con aquellos pares que comparten sus mismos intereses académicos, formando grupos de estudio. Al respecto, los relatos evocan a Vygotsky (1995), quien asume el aprendizaje y el desarrollo como el resultante de las interacciones que suceden en contextos históricos, sociales y culturales: "Yo a veces me reúno con mis amigos y a veces me ayudan" (EX9O23EN).

Los estudiantes señalan condiciones del entorno que favorecen o no el desarrollo de sus estudios; los textos reflejan que entornos como su barrio no son muy adecuados debido al ruido intenso o el escándalo. Estos estudiantes en condiciones en contra como las descritas se esfuerzan, superan obstáculos e influencias negativas como la de las pandillas o las drogas. Para ilustrarlo, obsérvese el próximo fragmento: "El ruido de las motos y autos, y a veces el alto volumen de los equipos de sonido que generan que me desconcentre en mis estudios" (EX9018EN).

Vale la pena señalar que ni las características personales, la cultura, ni el contexto y las características escolares influyen directamente en la motivación del individuo, de su pensamiento y su conducta hacia la consecución de sus logros, sino más bien la regulación activa de estos aspectos, es decir, a la autorregulación de la motivación y la conducta. (Alcántara \& Bernal, 2012; Bandura, 1991; Pintrich, 1999). En la Figura 2 se representan de forma metafórica estos hallazgos del sujeto cognoscente. 


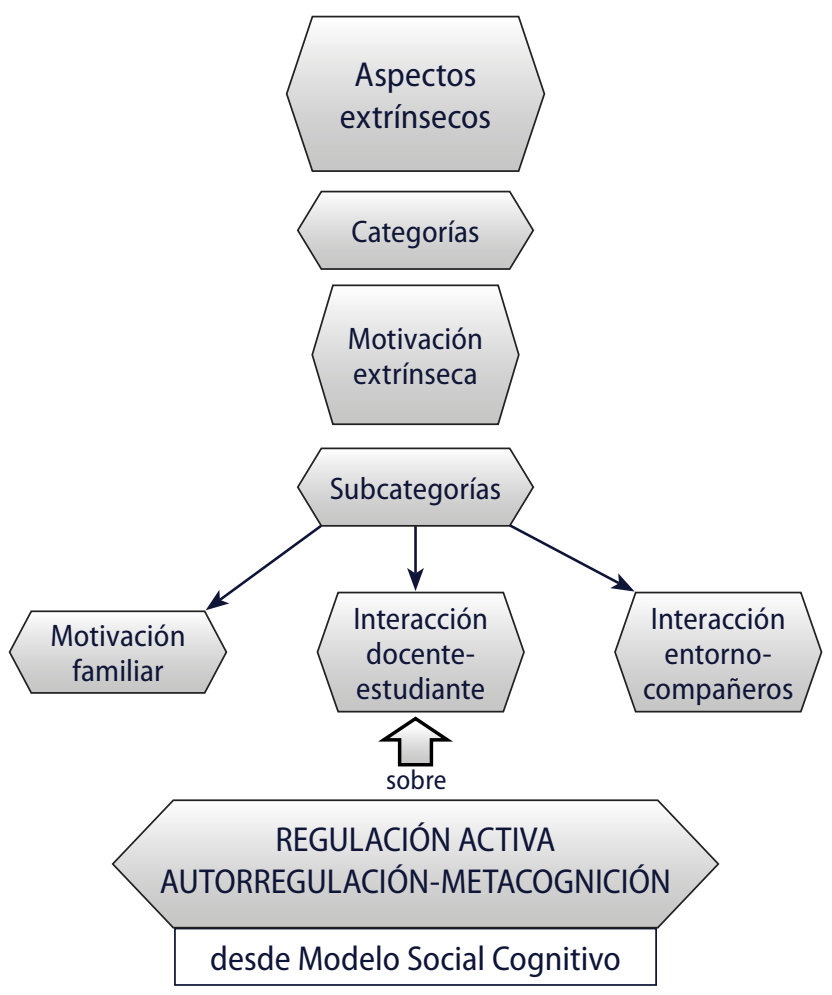

Figura 2. Relación entre componentes motivacionales extrínsecos y el constructo aprendizaje autorregulado

Fuente: Elaboración propia.

\subsection{Estrategias y características de aprendizaje}

Por último, el tercer aspecto referido a las estrategias y características de aprendizaje se constituyó gracias a las categorías y subcategorías registradas en la Tabla 4.

La constitución de este aspecto deja al descubierto diversas estrategias empleadas por los participantes, que han sido estudiadas por varios autores (Efklides, 2009; Ellis, Denton \& Bond, 2014; Roux \& Anzures, 2015; Sánchez, Castaño \& Tamayo, 2015; Yamac \& Ocak, 2013). 


\section{Tabla 4. Categorías y subcategorías del componente características y estrategias de aprendizaje}

\begin{tabular}{|c|c|c|}
\hline Aspecto central & Categoría & Subcategoría \\
\hline \multirow{10}{*}{$\begin{array}{c}\text { Estrategias y } \\
\text { características de } \\
\text { aprendizaje }\end{array}$} & \multirow{5}{*}{$\begin{array}{l}\text { Estrategias } \\
\text { actitudinales y } \\
\text { afectivas }\end{array}$} & Estrategias afectivas \\
\hline & & Estrategias de control del contexto \\
\hline & & Estrategias de procesamiento de información \\
\hline & & Estrategias de búsqueda y selección de información \\
\hline & & Estrategias de adquisición de conocimiento \\
\hline & \multirow{3}{*}{$\begin{array}{l}\text { Metacognición } \\
\text { y características } \\
\text { autorregulatorias }\end{array}$} & Comprender, no memorizar \\
\hline & & Conocimiento de cómo aprenden \\
\hline & & Autoadministración \\
\hline & \multirow{2}{*}{$\begin{array}{c}\text { Resolución de } \\
\text { problemas }\end{array}$} & Solucionan problema \\
\hline & & No solucionan problema \\
\hline
\end{tabular}

Fuente: Elaboración propia.

Estrategias afectivas fue la primer subcategoría identificada en comentarios como: "Un lugar tranquilo y mucho material de investigación" (EX9015EN); "Investigo los nuevos temas, pero a los que más me agrada les dedico más tiempo, como es la ciencia” (EX90315EN).

Se develan además estrategias afectivas y motivacionales que básicamente consisten en procedimientos de orden afectivo que los estudiantes utilizan, como "un lugar tranquilo", "escuchar música”, entre otros. Nótese que lo anterior implica conocimiento sobre la forma particular de llevar a cabo tales aprendizajes, y poner en disposición acciones para lograrlo. Sin lugar a dudas, estas son muestras de características autorregulatorias y de metacognición.

En el caso de las estrategias afectivas interpersonales emergen de textos como: "Por ejemplo, nos ponen una actividad y mis compañeros no entienden, yo trato de ayudar a mis compañeros y así sé que puedo aprender” (EX9024GF).

En el marco de la inteligencia interpersonal, este estudiante comprende a los demás, trabaja cooperativamente con sus pares, pero también comprende que con ello facilita y asegura su propio aprendizaje.

Posteriormente se destaca la subcategoría estrategias de control del contexto, referida a aspectos como las condiciones ambientales y la disposición de material de 
estudio. El espacio donde llevan a cabo los rituales de aprendizaje son, según sus relatos: "Mi cuarto, por su comodidad" (EX9023EN); "En el patio o el piso de la sala" (EX9022EN). Para la gran parte de este grupo de diez estudiantes, el silencio de la habitación y el hogar constituyen el lugar y condiciones ideales que favorecen su aprendizaje. Adviértase nuevamente la consciencia que reflejan en cuanto a las condiciones contextuales que posee cada uno de ellos para propiciar su propio aprendizaje.

A continuación, en la subcategoría estrategias de procesamiento de información destacan las estrategias atencionales, al hacer uso de diversos modos a fin de dirigir su atención hacia el logro deseado: "Aplicándome en clases, prestando atención a lo que expliquen" (EX9017EN); "Mediante participar de lo que el profesor pregunte" (EX9018EN).

Se reitera que los estudiantes han identificado los mecanismos que les permiten comprender un contenido, y más aún, se percibe el control de su atención a fin de lograr este cometido. Ponen en evidencia autoconocimiento y autocontrol, aspectos indisolublemente asociados a la autorregulación y a la metacognición (Flavell, 1976; Zimmerman, 2002; Efklides, 2009). De igual manera, la subcategoría estrategias de búsqueda y selección de información se estableció a partir de textos donde los estudiantes señalan hacer uso de la biblioteca o Internet a fin de cumplir con los compromisos o hallar respuestas a dudas o actividades escolares.

En la subcategoría estrategias de adquisición de conocimiento se agruparon los textos que señalaban a todas aquellas estrategias usadas para lograr que un nuevo concepto logre formar parte de la estructura mental cognitiva de los estudiantes, tales como: "Leer, hacer resúmenes, mapas conceptuales" (EX9021EN); "Mirando las tareas que hay, haciéndolas el día que las pongan, así no toquen el día siguiente, anotando en una agenda si hay evaluaciones" (EX9023EN).

Estas estrategias constituyen un conjunto de acciones que responden a un plan previo que realizan para lograr aprender; con ello los estudiantes ponen en evidencia, mediante sus textos, el hecho de que las estrategias de aprendizaje son un constructo importante en el aprendizaje autorregulado.

En cuanto a la segunda categoría, metacognición y características autorregulatorias, se constituyó primero por una razón pragmática: los relatos de los participantes revelan de forma implícita la estrecha relación entre estos dos constructos; segundo, los autores referenciados, como Efklides (2009), Pintrich (1999) y Zimmerman (2002) también lo hacen. En esta categoría se incluyeron las subcategorías comprender, no memorizar; conocimiento de cómo aprenden, y autoadministración. Se visualizan procesos metacognitivos en textos como: 
O sea, yo prácticamente, yo siempre, cuando terminan las clases, trato de entender lo que di, cuando ya entiendo yo hablo sobre este tema así sea sola [riéndose]. Y por ejemplo, nos ponen una actividad y mis compañeros no la entienden, yo trato de ayudar a mis compañeros y así sé que puedo aprender más. Pues a mí no me sirve repetir de memoria, sino hablar y entenderlo, porque de nada me sirve que yo me sepa de memoria pero ni siquiera lo entienda. (EX9024GF)

Se observa que el estudiante es consciente de que comprender y no sólo memorizar es fundamental para lograr el aprendizaje, y además, de cómo es posible que tal situación ocurra en su propio ser; esto es metaconocimiento, proceso de alto nivel de consciencia y supervisión activa a fin de gestionar procesos cognitivos (Efklides, 2009; Salmerón \& Gutiérrez, 2012; Zimmerman, 2002). Agréguese cómo ejerce control sobre sus propios mecanismos de aprendizaje: "hablo de ese tema, trato de ayudar a mis compañeros".

En la subcategoría autoadministración se incluyen relatos que describen cómo regulan su tiempo, disciplina y atención, estableciendo prioridades y logrando un sentido de compromiso, más allá de la tarea, con su propio aprendizaje. Es el tipo de estudiante reflexivo que suele investigar por su cuenta lo que los maestros exponen, para saber si es cierto (Sánchez \& Andrade, 2014).

Los mecanismos autorregulatorios y metacognitivos se evidencian en la categoría resolución de problemas, y es que éstos se utilizan para lograr, según Sánchez y Andrade (2014), "mejores desempeños en solución de problemas" (p. 1156).

Por otro lado, interpretar la información obtenida al invitar a los estudiantes a explicar un ejercicio relacionado con una temática evaluada recientemente por su docente de Ciencias (sobre calcular estados de oxidación de los elementos que forman parte de un compuesto), intentando percibir la manera como cada estudiante trató de solucionarlo, así como las estrategias de las que echan mano para darle solución, permitió dar cuenta de procesos de autorregulación y metacognición. Para resolver este ejercicio, haber comprendido conceptos tales como oxidación, electronegatividad, iones, equilibrio de carga, entre otras temáticas propias del plan de contenidos de octavo y noveno grado, es fundamental.

Por consiguiente, la categoría resolución de problemas se constituyó a partir de las subcategorías solucionan problema y no solucionan problema. En los textos que posibilitaron esa construcción se identificaron varios indicadores relacionados con esta competencia científica, que permitió una mejor comprensión de la manera como ponen en funcionamiento sus herramientas cognitivas los estudiantes a la hora de 
interpretar y solucionar un problema, haciendo uso de ideas previas y empleando un lenguaje formal. En contraste, en la subcategoría no solucionan problema se verificaron indicadores como el no uso de lenguaje formal y tendencia a ser algebraicos, pero sin entender con claridad los conceptos implicados. En efecto, algunos estudiantes manejaron estrategias metacognitivas, sin embargo, una parte de ellos, los que no pudieron resolver el ejercicio, no utilizaron estrategias que les ayudaran a solucionarlo o las que utilizaron no fueron eficaces, porque no les permitió lograr el éxito de la actividad.

Se pudo percibir que las estrategias de aprendizaje utilizadas por los estudiantes participantes corresponden a un carácter multidimensional (cognitivo, metacognitivo, motivacional), que los estudiantes han adquirido. Sin embargo, tal como lo señala García (2012), "dada su naturaleza flexible y posibilidad de transferencia, son los mismos estudiantes los que deciden cómo y dónde pueden aplicarlas” (p. 206). Y es precisamente decidir cómo y cuándo deben ser aplicadas y el control progresivo de las estrategias lo que implica autorregulación (Efklides, 2009; Pintrich, 1999; Zimmerman, 2002). Tal como señalan Panadero y Alonso-Tapia (2014), "la activación de las estrategias adecuadas depende de la autorregulación, siendo ésta una capacidad fundamental para que los alumnos tengan éxito" (p. 450). De igual forma, adquirir consciencia sobre el uso que se hace de éstas es un aspecto esencial, en la medida que si no hay consciencia, no podrá mejorarse en su aplicación, lo cual se refiere a metacognición (García, 2012; Yamac \& Ocak, 2013).

Después de un diálogo entre las voces de participantes, teorías y autores sobre las características de los estudiantes exitosos, mediante una descripción cuidadosa de cada una de las categorías emergidas, fue posible acercarse al "eidos", como diría Martínez (2011, p.169), de este fenómeno. Proponemos la Figura 3 para, de alguna manera, intentar trascender los límites descriptivos o sustantivos hacia la formalidad. De allí que en una metáfora se representa la acción de la autorregulación y de la metacognición a modo de doble hélice que se imbrica mediante los aspectos motivacionales tanto internos como externos. 


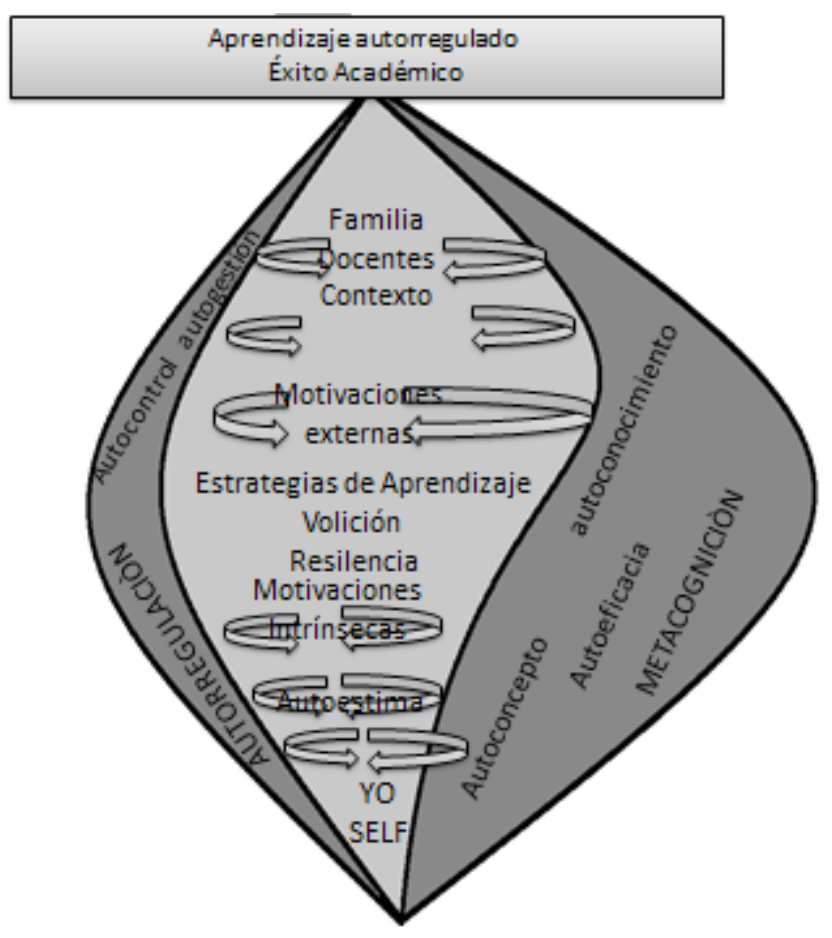

\section{Figura 3. Constructo multidimensional de la autorregulación- metacognición en el éxito académico de los estudiantes}

Fuente: Elaboración propia.

Adviértase que la base de la figura, su génesis, es el Yo, de quien finalmente pende su control y desarrollo. En el extremo superior, como consecuencia de las interrelaciones entre los aspectos motivacionales, la autorregulación y la metacognición ejercidas y desarrolladas por el Yo, se sitúa el aprendizaje autorregulado, y con ello, el éxito académico de los estudiantes. En el interior se sitúan componentes intrínsecos y extrínsecos que se presentan ante los estudiantes a modo de peldaños; sin embargo, no son para nada estáticos, sino que se encuentran en una constante interacción que fusiona y solapa un extremo (autorregulación) y otro (metacognición), puesto que tales componentes están estrechamente relacionados, tal como se halló a través de la información aportada por los estudiantes y por los autores referenciados. 


\section{Consideraciones finales}

A partir de este diálogo entre los textos de los estudiantes, las interpretaciones del sujeto cognoscente y la posterior contrastación con los autores, se atisban las siguientes aproximaciones:

- En primera instancia se apunta hacia las principales características percibidas en los estudiantes exitosos; se comprendió que a la luz de sus subjetividades y tal como señalan las teorías y autores referenciados, el éxito académico corresponde con aspectos intrínsecos como: motivaciones intrínsecas y autoconcepto, y aspectos externos como: motivaciones extrínsecas, conformadas por la influencia de la familia, en la cual sobresale la motivación de la madre, la influencia de los docentes, del entorno y de los compañeros. Se percibió que existe una gran variedad de razonamientos, estrategias y características. Ahora bien, los estudiantes coinciden al señalar que la disciplina y la responsabilidad los distingue del resto de sus compañeros, características que se corresponden con la autorregulación, la metacognición y, en particular, la autoadministración.

- Se comprendió que la metacognición se vincula estrechamente a la autorregulación académica. En tanto que las fronteras entre un concepto y otro resultan difusas, se ha tratado convenientemente de hablar del constructo autorregulación-metacognición. Pudo verificarse cómo los mecanismos metacognitivos y autorregulatorios se imbrican en una red compleja de interacciones entre aspectos motivacionales de carácter intrínseco y extrínseco. Además, se evidenció que los estudiantes exitosos aplican estrategias de aprendizaje de manera frecuente y efectiva.

- En tercer lugar, algunas inferencias desarrolladas a partir de los hallazgos apuntan a orientar el quehacer pedagógico de los docentes de Ciencias Naturales, a fin de promover aprendizajes autorregulados en un mayor número de estudiantes. Por consiguiente, los docentes de Ciencias han de promover estrategias de enseñanza diversificadas que atiendan a diversos estilos de aprendizaje, y valorar la capacidad de involucrar de forma creativa a sus estudiantes.

- Surgen interrogantes que se proyectan como una continuación del trabajo, tales como: ¿Qué características deben tener las estrategias usadas por los docentes de Ciencias para favorecer el aprendizaje autorregulado?; iqué estrategias y acciones pueden promover que los estudiantes logren autorregular su aprendi- 
zaje en la internalización de conceptos propios de las Ciencias?; ¿cómo pueden los docentes de Ciencias fomentar el uso de estrategias metacognitivas en sus aprendices?, y ¿cómo dar cuenta de las estrategias metacognitivas en las pruebas de evaluación? Finalmente, se advierte que las pretensiones del estudio, debido a su naturaleza interpretativa, son locales, y cualquier ambición de universalizarlos debe ser contextualizada en la realidad de los sujetos participantes del estudio y de la institución educativa del municipio de Malambo donde se desarrollaron.

\section{Lista de referencias}

Alcántara, V., \& Bernal, S. (2012). El aprendizaje autorregulado y su relación con la motivación en los estudiantes universitarios. DIDAC, Motivación y Docencia, 59, 4-10. Recuperado de http://revistas.ibero.mx/didac/uploads/volumenes/1/ $\mathrm{pdf} / 59 . \mathrm{pdf}$

Bandura, A. (1991). Social Cognitive Theory of Self-Regulation. Organizational Behavior and Human Decision Process, 50, 448-287. Recuperado de http://www. uky.edu/ eushe2/BanduraPubs/Bandura19910BHDP.pdf

Becerra, C., \& Morales, M. (2015). Validación de la Escala de Motivación de Logro Escolar (EME-E) en estudiantes de bachillerato en México. Innovación Educativa, 15(68), 135-153. Recuperado de http://www.innovacion.ipn.mx/Revistas/Documents/Revistas-2015/I-E-68/IE-68.pdf

Berger, J.-L. (2012). Motivational Beliefs and Self-regulated Learning in Low Vocational Training Track Students. Journal of Educational and Developmental Psychology, 2(1), 37-48. doi:http://dx.doi.org/10.5539/jedp.v2n1p37

Boekaerts, M. (1999). Motivated learning: Studying student * situation transactional units. European Journal of Psychology of Education, 14(1), 41-55. Recuperado de https://www.researchgate.net/profile/Monique_Boekaerts/publication $/ 232597700$ Context_sensitivity_Activated_motivational beliefs current_concerns and_emotional_arousal/links/56a4f75008aef91c8c14c736. pdf

Coffey, A., \& Atkison, P. (2003). Encontrar el sentido a los datos cualitativos: Estrategias complementarias de investigación. Colombia: Facultad de Enfermería-Universidad de Antioquia. Recuperado de http://www.fceia.unr.edu.ar/geii/maes- 
tria/2014/DraSanjurjo/8mas/Amanda\%2oCoffey,\%2oEncontar\%2oel\%20 sentido\%20a\%2olos\%2odatos\%2ocualitativos.pdf

Creswell, J. (1994). Diseño de Investigación. A proximaciones cualitativas y cuantitativas. Argentina: Universidad de Buenos Aires. Recuperado de http://www.catedras. fsoc.uba.ar/ginfestad/biblio/1.2.\%20Creswell.\%20A\%2oqualit....pdf

Efklides, A. (2009). The role of metacognitive experiences in the learning process. Psicothema, 21(1), 76-82.

Ellis, A. Denton, D., \& Bond, J. (2014). An analysis of research on metacognitive teaching strategies. Procedia-Social and Behavioral Sciences, 116, 40154024. Recuperado de http://www.sciencedirect.com/science/article/pii/ $\underline{S_{1} 877042814009008}$

Flavell, J. H. (1976). Metacognitive aspects of problem solving. En L. B. Resnick (Ed.), The nature of intelligence (pp. 231-325). Estados Unidos: Erlbaum.

García, M. (2012). La autorregulación académica como variable explicativa de los procesos de aprendizaje universitarios. Profesorado. Revista de currículum y formación del profesorado, 16(1), 203-221. Recuperado de http://www.ugr. es/ recfpro/rev161ART12.pdf

Gómez, M. (2003). Algunos Factores que influyen en el éxito académico de los estudiantes universitarios en el área de Química (Tesis doctoral). Departamento de Didáctica de las Matemáticas y de las Ciencias Experimentales, Universidad Autónoma de Barcelona, España. Recuperada de http://ddd.uab.cat/pub/tesis/2003/ tdx-1202103-144528/mgmide1.pdf

González, M. (2013). Los estilos de enseñanza y aprendizaje como soportes de la actividad docente. Revista Estilos de Aprendizaje, 11(11). Recuperado de http://www2. uned.es/revistaestilosdeaprendizaje/numero 11/articulos/articulo_04.pdf

Hernández, R., Fernández, C., \& Baptista, M. (2010). Metodología de la investigación ( $5^{\mathrm{a}}$ ed.). México: McGraw-Hill/Interamericana.

Husserl, H. (1974). Ideas relativas a una fenomenología pura y una filosofía fenomenológica. México: Fondo de Cultura Económica.

Instituto Colombiano para el Fomento de la Educación Superior. (2016). SABER $3^{\circ}, 5^{\circ} y$ $9^{\circ}$. Resultados nacionales 2009-2014 (Informe Nacional). Recuperado de http:// www.icfes.gov.co/docman/investigadores-y-estudiantes-de-posgrado/informes-de-resultados-evaluaciones-nacionales/informes-resultados-saber3-5-y-9/2323-resultados-nacionales-saber-30-50-y-90-2009-2014/file?forcedownload $=1$ 
Lanz, M. Z. (2006). El Aprendizaje autorregulado. Enseñar a aprender en diferentes entornos educativos (Colección Ensayos y Experiencias). Argentina: Noveduc.

Martínez, M. (2011). Comportamiento humano. Nuevos métodos de investigación ( $2^{\mathrm{a}}$ ed.). México: Trillas.

Maturana, H. (2002). Emociones y lenguaje en educación y política. Chile: JC Sáez.

Núñez, J. C., Amieiro, N., Álvarez, D., García, T., \& Dobarro, A. (2015). Escala de evaluación de la autorregulación del aprendizaje a partir de textos (ARATEX-R). European Journal of Education and Psychology, 8(1), 9-22. Recuperado de http:// www.redalyc.org/pdf/1293/129343965002.pdf

Panadero, E., \& Alonso-Tapia. (2014). ¿Cómo autorregulan nuestros alumnos? Revisión del modelo cíclico de Zimmerman sobre autorregulación del aprendizaje. Anales de Psicología, 30(2), 450-462. Recuperado de http://revistas.um.es/analesps/article/view/analesps.30.2.167221

Pintrich, P. (1999). The role of motivation in promoting and sustaining self-regulated learning. International Journal of Educational Research, 31, 459-470.

Rojas, B. (2014). Investigación Cualitativa. Fundamentos y Praxis ( $3^{\mathrm{a}} \mathrm{ed}$.). Venezuela: Universidad Pedagógica Experimental.

Rosario, P., Pereira, A., Högemann, J., Nunes, A. R., Figueiredo, M., Núñez, J. C., Fuentes, S., \& Gaeta, M. L. (2014). Autorregulación del Aprendizaje: una revisión sistemática en revistas de la base SciELO. Universitas Psychologica, 13(2), 781-798. Recuperado de http://revistas.javeriana.edu.co/index.php/revPsycho/article/viewFile/4487/8540

Roux, R., \& Anzures, E. (enero-abril, 2015). Estrategias de aprendizaje y su relación con el rendimiento académico en estudiantes de una escuela privada de educación media superior. Revista Electrónica Actualidades Investigativas en Educación, 15(1), 1-16. doi:http://dx.doi.org/10.15517/aie.v15i1.17731

Ryan, R., \& Deci, E. (200o). La Teoría de la Autodeterminación y la Facilitación de la Motivación Intrínseca, el Desarrollo Social, y el Bienestar. American Psychologist, 55(1), 68-78. Recuperado de http://www.davidtrotzig.com/uploads/articulos/2000_ryandeci_spanishampsych.pdf

Salmerón, H., \& Gutiérrez, C. (2012). La competencia de aprender a aprender y el aprendizaje autorregulado. Posicionamientos teóricos. Profesorado. Revista de currículum y formación del profesorado, 16(1). Recuperado de http://www.ugr. es/ recfpro/revi61ART1.pdf

Sánchez, L., \& Andrade, R. (2014). Inteligencias múltiples y estilos de aprendizaje. Mé- 
xico: Alfaomega.

Sánchez, J., Castaño, O., \& Tamayo, O. (2015). La argumentación metacognitiva en el aula de ciencias. Revista Latinoamericana de Ciencias Sociales, Niñez y Juventud, 13(2), 1153-1168. Recuperado de http://revistalatinoamericanaumanizales. cinde.org.co/wp-content/uploads/2015/o8/La-argumentaci\% $\mathrm{C}_{3} \% \mathrm{~B}_{3}$ _ vol13n2a42.pdf

Serbia, J. (2007). Diseño, muestreo y análisis en la investigación cualitativa. Hologramática, $3(7), 123-146$. Recuperado de http://www.cienciared.com.ar/ra/usr/3/206/ n7_vol3pp123_146.pdf

Strauss, A., \& Corbin, J. (2002). Bases de la Investigación Cualitativa. Técnicas y Procedimientos para desarrollar la Teoría Fundamentada. Colombia: Facultad de Enfermería-Universidad de Antioquia Recuperado de https://diversidadlocal.files.wordpress.com/2012/og/bases-investigacion-cualitativa.pdf

Urbina, J. E. (2012). La pasión de aprender. El punto de vista de los estudiantes universitarios (Tesis doctoral). Centro de Estudios Avanzados en Niñez y Juventud. Universidad de Manizales-Centro Internacional de Educación y Desarrollo Humano, Colombia. Recuperada de http://biblioteca.clacso.edu.ar/Colombia/alianza-cinde-umz/20130402114226/TesisJesusUrbinaCardenas.pdf

Vasilachis, I. (Coord.). (2006). Estrategias de investigación cualitativa. España: Gedisa. Recuperado de http://biblioteca.clacso.edu.ar/Colombia/alianza-cindeumz/20130402114226/TesisJesusUrbinaCardenas.pdf

Vygotsky, L. (1995). Pensamiento y Lenguaje. Teoría del desarrollo cultural de las funciones psíquicas. Buenos Aires: Fausto. Recuperado de http://biblioteca.iesit. edu.mx/biblioteca/Loo947.PDF

Weiner, B. (1985). An Attributional Theory of Achievement Motivation and Emotion. Psychological Review, 92(4), 548-573.

Weiner, B. (2000). Intrapersonal and Interpersonal Theories of Motivation from Attributional Perspective. Educational Psychology Review, 12(1), 1-14. Recuperado de https://www.researchgate.net/publication/239768172 Intrapersonal and Interpersonal Theories of Motivation from an Attribution Perspective

Yamac, H., \& Ocak, G. (2013). Examination of the Relationships between fifth graders' Self-Regulated Learning Strategies, Motivational Beliefs, Attitudes, and Achievement. Educational Sciences: Theory of Practice, 13(1), 390-387. Recuperado de http://files.eric.ed.gov/fulltext/EJ1016657.pdf 
Zimmerman, B. J. (2002). Becoming a Self-Regulated Learner: An Overview. Theory Into Practice, 41(2), 64-70. Recuperado de: http://saifulislam.com/wp-content/uploads/2016/10/Becoming-a-Self-Regulated-Learner-An-Overview. pdf

Zimmerman, B.J., \& Moylan, A. R. (2009). Self-regulation: Where metacognition and motivation intersect. En D.-J. Hacker, J. Dunlosky \& A. C. Graesser (Eds.), Handbook of Metacognition in Education (pp. 299-315). Nueva York: Routledge. 